\title{
AVALIAÇÃO DA COMPOSIÇÃO QUÍMICA DOS EXTRATOS DA RAIZ DE Derris urucu OBTIDOS POR $\mathrm{CO}_{2}$ SUPERCRÍTICO
}

\author{
ALMEIDA, O. ${ }^{1}$, MACHADO, N. T. ${ }^{1}$, ARAÚJO, M. E. ${ }^{1}$, MOREIRA, R. F. ${ }^{1}$, SOUZA FILHO, A. P. \\ S. $^{2}$, LOPES, J. D. N. F. ${ }^{3}$, CHAGAS, B. R. ${ }^{3}$, MARTINS, L. R. S. ${ }^{3}$ \\ ${ }^{1}$ Faculdade de Engenharia Química, Instituto de Tecnologia, Universidade Federal do Pará \\ ${ }^{2}$ EMBRAPA-PA Amazônia Oriental \\ ${ }^{3}$ Faculdade de Química, Instituto de Ciências Exatas e Naturais, Universidade Federal do Pará \\ E-mail: ossalin@ufpa.br
}

\begin{abstract}
RESUMO - Timbó é o nome pelo qual são conhecidas, na Amazônia, inúmeras plantas de cultura pré-colombiana. A palavra timbó é de origem tupi ti (suco), e mb (cobra), significando sumo de cobra, serve também para designar plantas pertencentes a diversas famílias botânicas, principalmente Sapindaceae e Fabaceae, as quais apresentam propriedades ictiotóxicas. Este trabalho teve como objetivo o estudo da composição química dos extratos da raiz de Derris urucu obtidos por $\mathrm{CO}_{2}$ supercrítico. Foram avaliados os teores de rotenona e de deguelina em três condições experimentais $\left(\mathrm{C} 1: \mathrm{T}=35^{\circ} \mathrm{C}, \mathrm{P}=120,220\right.$ e 300 bar; $\mathrm{C} 2: \mathrm{T}=40^{\circ} \mathrm{C}, \mathrm{P}=$ 120,220 e 300 bar e $\mathrm{C} 3: \mathrm{T}=60^{\circ} \mathrm{C}, \mathrm{P}=120,220$ e 300 bar). Os teores médios de rotenona e deguelina da composição química dos extratos obtidos por percolação e por SFE foram de 14,01 e 8,21\% (percolação, $\mathrm{T}=\mathrm{amb}, \mathrm{t}=96 \mathrm{~h}$ ) e de 23,30 e $18,49 \%\left(\mathrm{~T}=60^{\circ} \mathrm{C}, \mathrm{P}=300\right.$ bar e $\left.\mathrm{t}=90 \mathrm{~min}\right)$, correspondentes a 93,16 e $73,97 \%$ do teor total contido na amostra in natura.
\end{abstract}

\section{INTRODUÇÃO}

Nas raízes de Derris spp. e Lonchocarpus spp., encontram-se seis substâncias denominadas rotenóides (1- $\alpha$-toxicarol, eliptona, sumatrol, malacol, rotenona e deguelina). A rotenona e a deguelina são os mais explorados em estudos e pesquisas.

A extração por fluido supercrítico é uma técnica que pode substituir os processos tradicionais de extração (destilação, extração líquido-líquido e extração sólido-líquido). A maior parte dos compostos orgânicos que apresentam interesse ao ser humano, em sua forma natural, encontra-se em matrizes biológicas complexas. Com o objetivo de separar estes compostos da sua forma original, eles são colocados em contato com outras fases. Estas fases podem ser gás-liquido, vapor-líquido, líquidolíquido, sólido-gás ou sólido-líquido.

A motivação principal deste trabalho está na possibilidade do enriquecimento e/ou separação da rotenona e deguelina para aumentar o seu valor agregado. De acordo com a revisão da literatura verificou-se a carência existente no que diz respeito a estudos que exploram a união de duas áreas do conhecimento que englobam a extração e a concentração de princípios ativos em um único processo. 


\section{MATERIAIS E MÉTODOS}

\subsection{Coleta do Material Vegetal}

As raízes de Timbó (Derris urucu), foram coletadas no Banco Ativo de Germoplasma Timbó da Embrapa Amazônia Oriental $\left(01^{\circ} 25^{\prime} 45^{\prime}\right.$ ' S e $48^{\circ} 24^{\prime} 09^{\prime \prime} \mathrm{W}$, a $22 \mathrm{~m}$ de altitude). A identificação botânica foi realizada no Herbário do Centro de Pesquisa Agroflorestal da Amazônia Oriental da EMBRAPA (IAN). As coletas foram realizadas conforme a Instrução Normativa № 154, de 01 de Março de 2007 do Instituto Brasileiro do Meio Ambiente e dos Recursos Naturais Renováveis IBAMA.

\subsection{Pré-Tratamento e Caracterização do Material Vegetal}

A preparação da amostra (raízes de Timbó) consistiu de lavagem, secagem à temperatura ambiente durante 72 h, secagem em estufa com circulação de ar (MARCONI, modelo 035) a $40 \pm 2$ ${ }^{\circ} \mathrm{C}$, durante sete dias, com pesagens a cada 24 horas.

A moagem foi realizada em moinho de facas (WILLEY, Modelo WLS-3004) e acondicionado em sacos plásticos. A distribuição granulométrica foi realizada através de um agitador magnético de peneiras (BERTEL, modelo 1868), durante 30 minutos com agitação no nível 5, tendo sido utilizadas peneiras da série padrão Tyler de 9-48 mesh (MENTOR, modelo RX-29-10).

O diâmetro médio da partícula foi determinado de acordo com a metodologia do diâmetro médio de Sauter (FIORI et al., 2008), conforme descrito na Equação 1.

$$
d_{m}=\frac{\sum_{i=1}^{n} \cdot\left(m_{i} \cdot d_{i}^{3}\right)}{\sum_{i=1}^{n} \cdot\left(m_{i} \cdot d_{i}^{2}\right)}
$$

Onde, $d_{m}$ é o diâmetro médio $(\mathrm{mm}), \mathrm{m}_{i}$ é a massa retida na $i$-ésima peneira $(\mathrm{g})$ e $d_{i}$ é a abertura nominal da $i$-ésima peneira $(\mathrm{mm})$. Em todas as extrações, foram utilizadas partículas de diâmetro 24 mesh $(0,71 \mathrm{~mm})$.

A determinação do diâmetro médio geométrico da partícula $\left(d_{g}\right)$ foi determinada de acordo com o método ASAE S319.3 (ASAE Standards, 1998), conforme a Equação 2.

$d_{g}=\log ^{-1} \cdot\left[\frac{\sum_{i=1}^{n} \cdot\left(W_{i} \cdot \log \cdot \bar{d}_{i}\right)}{\sum_{i=1}^{n} \cdot W_{i}}\right]$

Onde, $\bar{d}_{i}\left(\bar{d}_{i}=\left(d_{i} . d_{i+1}\right)^{0,5}\right)$ é a abertura nominal da $i$-ésima peneira $(\mathrm{mm}) ; d_{i+1}$ é a abertura nominal da peneira maior que a $i$-ésima peneira $(\mathrm{mm}) ; W_{i}$ é a massa do material retido na $i$-ésima peneira $(\mathrm{g})$. 


\subsection{Obtenção dos Extratos Vegetais}

Com o objetivo de comparar os resultados de rendimentos e a composição química (teores de rotenona e de deguelina) dos extratos da raiz de Derris urucu, foram utilizados dois processos de extração: por percolação a frio e por fluido supercrítico $\left(\mathrm{CO}_{2}\right)$.

Percolação: $\mathrm{O}$ extrato foi obtido em um percolador de aço inox $(5 \mathrm{~L})$, contendo $500 \mathrm{~g}$ de material seco e moído (raízes de Timbó), foram feitas duas extrações com 2 L de metanol (P.A, Nuclear, Brasil) a cada 48 horas. As frações foram reunidas, o solvente foi removido em evaporador rotativo (HEIDOLPH, Laborota 4000), a pressão reduzida na temperatura de $50^{\circ} \mathrm{C}$ até, aproximadamente, $1 / 5$ do seu volume. $\mathrm{O}$ extrato bruto metanoico (DU-MeOH) foi transferido para frascos de vidro âmbar e armazenado em freezer doméstico (Cooler H-300 Eletrolux) a -10 C.

Extracão com Fluido Supercrítico: Os extratos foram obtidos na Planta Piloto de Extração Supercrítica do Laboratório de Operações de Separação (LAOS/FEQ/UFPA). A representação esquemática do sistema é apresentada na Figura 1.

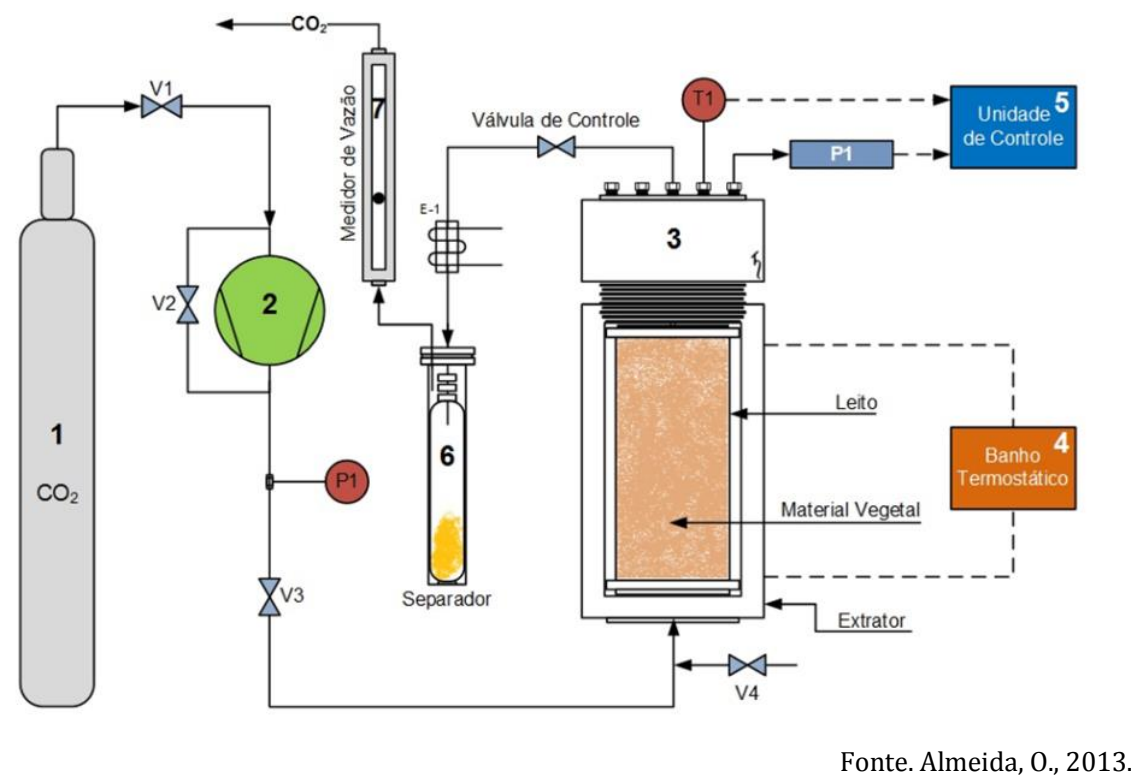

Figura 1- Fluxograma simplificado da unidade de extração supercrítica (LAOS/FEQ/UFPA).

A parte utilizada da planta consiste de: (1) cilindro de $\mathrm{CO}_{2}(99,0 \%$, CARBOMAM, BelémPA; (2) compressor de membrana (Andreas Hoffer, Mod. MKZ 120-50, Alemanha), com capacidade de elevar a pressão de 60 a 350 bar, a uma vazão de até $70 \mathrm{~kg} / \mathrm{h}$; (3) recipiente de aço inox com capacidade para 1,0 L, com camisa de aquecimento (Metalwerkstatt, TUHH, Alemanha), usado como extrator; (4) banho termostático (Haake Mess-Technik GmbH, Mod. N3, Alemanha); (5) unidade eletro-eletrônica de controle. As condições operacionais para a avaliação do efeito da pressão e da 
temperatura sobre os diferentes sistemas de extrações são apresentadas na Tabela 1. Para cada extração, a massa de amostra foi fixada em $200 \mathrm{~g}$, sendo esta, suficiente para o preenchimento total do leito.

Tabela 1 - Condições experimentais para avaliar o rendimento da extração da raiz de Derris urucu usando $\mathrm{CO}_{2}$ supercrítico $\left(Q m=12 \mathrm{~L} \cdot \mathrm{min}^{-1}\right)$.

\begin{tabular}{|c|c|c|c|c|}
\hline Espécie & $\begin{array}{c}\text { Condições } \\
\text { Experimentais }\end{array}$ & $\mathbf{t}[\mathrm{min}]$ & $\mathrm{T}\left[{ }^{0} \mathrm{C}\right]$ & $\mathbf{P}[\mathrm{bar}]$ \\
\hline \multirow{3}{*}{$\begin{array}{l}\text { Derris urucu } \\
\quad \text { (raiz) }\end{array}$} & $\mathrm{C} 1$ & 60 & 35 & $\begin{array}{l}120 \\
220 \\
300\end{array}$ \\
\hline & C2 & 60 & 40 & $\begin{array}{l}120 \\
220 \\
300\end{array}$ \\
\hline & C3 & 60 & 60 & $\begin{array}{l}120 \\
220 \\
300\end{array}$ \\
\hline
\end{tabular}

Nesta etapa, foram realizadas nove extrações, divididas em três condições experimentais (C1, C2 e C3), a fim de avaliar o rendimento, a estabilidade, a seletividade e a composição química dos extratos da raiz de Timbó obtidos no processo de Extração por Fluido Supercrítico (SFE).

A massa de $\mathrm{CO}_{2}\left(\mathrm{MCO}_{2}\right)$ foi calculada com o auxílio da equação dos gases ideais a partir dos dados de volume (medidor de vazão), pressão e temperatura ambiente. Como estas medidas foram realizadas em intervalos de tempo, a média foi considerada como a vazão do solvente $(Q m)$ (Equação 3) para todos os experimentos e o desvio padrão como o erro.

$Q_{m}=\left(\frac{\Delta M_{C O 2}}{\Delta t}\right)$

As análises químicas dos extratos foram realizadas em um Cromatógrafo Líquido de Alta Eficiência SHIMADZU PROMINENCE, constituído de uma bomba solenóide com quatro canais (modelo LC-20AT), detector de arranjo de diodo (modelo SPD-M20A), degaseificador de membrana (modelo DGU-20A), auto-injetor de amostras (modelo SIL-20A), interface de comunicação (modelo CBM-20A) acoplado a um microcomputador Pentium IV com software de integração LC solution operando em comprimento de onda fixo de $293 \mathrm{~nm}$, sistema isocrático $\mathrm{H}_{2} \mathrm{O}$ (A) : ACN (B) $60 \%$ de B, sob vazão de $1 \mathrm{~mL} / \mathrm{min}$ onde foi empregada uma coluna Gemini

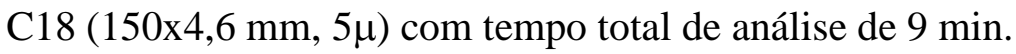




\section{RESULTADOS E DISCUSSÃO}

Neste trabalho, foram utilizados dois processos de extração para a obtenção dos extratos vegetais, por percolação a frio (DU-MeOH) e por $\mathrm{CO}_{2}$ supercrítico (SFE-DA). Os rendimentos médios em base seca para cada processo foram de: 11,30\% (96 h) e 2,64\% (3 h), respectivamente.

$\mathrm{Na}$ Tabela 2, são apresentados os resultados das SFE em três condições experimentais, C1, C2 e C3. Para todas as extrações foram usadas raiz de Derris urucu com as seguintes características: granulometria fixa de 24 mesh, correspondendo a um diâmetro médio de partícula de $0,71 \mathrm{~mm}$; densidade aparente de $0,4658 \mathrm{~g} . \mathrm{cm}^{-3}$; densidade real de $0,4865 \mathrm{~g} . \mathrm{cm}^{-3}$, porosidade do leito de 0,04251 , respectivamente, a uma vazão média de $12 \mathrm{~L} \cdot \mathrm{min}^{-1}$ de $\mathrm{CO}_{2} \mathrm{em} 60$ minutos de extração. A umidade do material vegetal foi de $4,98 \% \pm 0,01$ em base seca.

Tabela 2 - Avaliação preliminar das condições experimentais da extração por fluido supercrítico da raiz de Derris urucu $\left(\mathrm{t}=60\right.$ min e $Q_{m}=12$ L. $\left.\min ^{-1}\right)$.

\begin{tabular}{|c|c|c|c|c|c|c|c|}
\hline \multirow{2}{*}{$\begin{array}{c}\text { Condições } \\
\text { Experimentais }\end{array}$} & \multirow{2}{*}{$\begin{array}{c}\mathbf{P} \\
{[\text { bar }]}\end{array}$} & \multirow{2}{*}{ 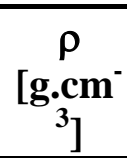 } & \multirow{2}{*}{$\begin{array}{l}\text { Red. } \\
{[\%]}\end{array}$} & \multicolumn{2}{|c|}{ Composição [\%] } & \multicolumn{2}{|c|}{$\begin{array}{c}\text { mg/100 Amostra } \\
\text { (b.s.) }\end{array}$} \\
\hline & & & & Rotenona & Deguelina & Rotenona & Deguelina \\
\hline \multirow{3}{*}{$\begin{array}{c}\mathbf{C 1} \\
\left(\mathrm{T}=35^{\circ} \mathrm{C}\right)\end{array}$} & 120 & 0,7685 & 0,29 & 0,68 & 1,28 & 3,91 & 7,35 \\
\hline & 220 & 0,8817 & 0,57 & 1,96 & 2,05 & 22,22 & 23,13 \\
\hline & 300 & 0,9296 & 0,61 & 2,42 & 1,76 & 29,58 & 21,56 \\
\hline \multirow{3}{*}{$\begin{array}{c}\mathbf{C 2} \\
\left(\mathrm{T}=40^{\circ} \mathrm{C}\right)\end{array}$} & 120 & 0,7198 & 0,58 & 1,17 & 1,68 & 17,00 & 24,32 \\
\hline & 220 & 0,8581 & 0,76 & 1,70 & 1,91 & 32,09 & 36,09 \\
\hline & 300 & 0,9109 & 0,95 & 2,25 & 2,05 & 53,23 & 48,58 \\
\hline \multirow{3}{*}{$\begin{array}{c}\mathbf{C 3} \\
\left(\mathrm{T}=60^{\circ} \mathrm{C}\right)\end{array}$} & 120 & 0,4359 & 1,03 & 1,45 & 1,52 & 39,01 & 40,90 \\
\hline & 220 & 0,7538 & 1,20 & 2,01 & 1,85 & 60,06 & 55,28 \\
\hline & 300 & 0,8303 & 1,40 & 5,30 & 3,45 & 172,88 & 112,53 \\
\hline
\end{tabular}

De acordo com os dados da Tabela 2, verifica-se que o aumento da temperatura a pressão constante, conduz a uma diminuição na densidade do $\mathrm{CO}_{2}$, diminuindo o poder de solvatação do solvente, por outro lado aumenta a pressão de vapor do soluto.

Segundo King e Bott (1993), o efeito combinado de ambos determinará o comportamento da variação da solubilidade com a temperatura e pressão. Esta tendência é observada quando analisamos as isotermas de rendimento da extração. Mediante a análise dos resultados dos rendimentos e composição química para os diferentes sistemas, o melhor resultado foi obtido na condição C3.

Mediante a avaliação dos resultados preliminares (Tabela 2), foram realizados novos experimentos, o tempo das extrações foi fixado em 90 minutos. Por motivo de segurança, devido a cristalização do extrato na entrada do separador, as coletas foram realizadas em intervalos de 15, 30, 60 e 90 minutos. 
Na Figura 2, são apresentados os teores médios de rotenona e deguelina nos extratos obtidos por percolação, $\mathrm{CO}_{2}$ pressurizado e na torta após extração supercrítica. $\mathrm{Na}$ extração por fluido supercrítico os teores médios de rotenona e deguelina, foram de 23,30\% e 18,49\% (90 minutos), correspondentes a $93,16 \%$ e $73,97 \%$ do teor total contido na amostra.

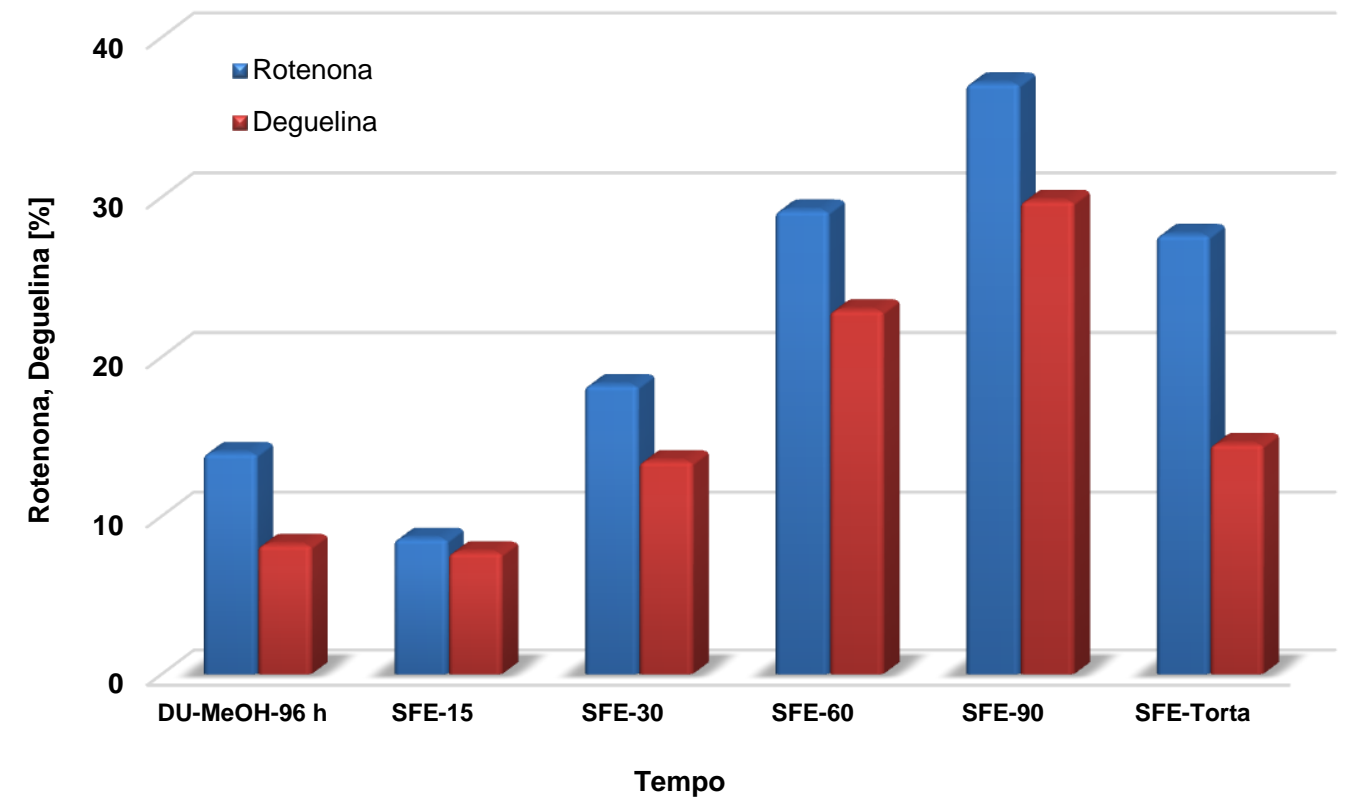

Figura 1 - Teores da rotenona e da deguelina nos extratos da raiz de Derris urucu, obtidos por percolação (DU-MeOH, $\left.\mathrm{T}_{\mathrm{Amb}}=28,5{ }^{\circ} \mathrm{C}, \mathrm{P}=1 \mathrm{~atm}\right)$ e $\mathrm{CO}_{2}$ pressurizado (SFE).

Em relação ao teor de rotenona, este trabalho obteve resultado superior aos obtidos por D' Andrea et. al., 2007, que avaliaram a influência dos parâmetros de processo da extração da rotenona da raiz de Derris elliptica (Wallich) Benth, por fluido supercrítico, onde conseguiram um rendimento de $11,25 \%$ de rotenona na temperatura de $60{ }^{\circ} \mathrm{C}$ e pressão de 440 bar.

\section{CONCLUSÃO}

$\mathrm{Na}$ extração por fluido supercrítico o rendimento em massa acumulada de extrato $(19,4820$ g) em 180 minutos de extração (duas extrações de 90 minutos), em comparação com a massa de extrato $(56,4818 \mathrm{~g})$ obtido por solvente (etanol) em 96 horas de extração.

Os teores de rotenona e deguelina do extrato metanoico (percolação) foram de 14,01 e $8,21 \%$ em 96 h de extração. Na extração por fluido supercrítico, os teores mais elevados de rotenona $(37,14 \%)$ e deguelina $(29,79 \%)$ foram conseguidos na fração coletada em 90 minutos, sendo estes 2,65 e 3,63 vezes maior em comparação com o extrato obtido por percolação.

Os teores médios de rotenona e deguelina, obtidos por fluido supercrítico em 90 minutos de extração foram de $23,30 \%$ e $18,49 \%$, correspondentes a $93,16 \%$ e $73,97 \%$ do teor total contido na amostra. 


\section{REFÊRENCIAS}

ASAE. Standards Method of Determining and Expressing Fineness of Feed Materials by Sieving. ASAE, S319.3, p.547, 1998.

BOOT, T. R. Fundamentals of Carbon Dioxide in Solvent Extraction. Chemical Industrial, v.19, n.6, p.394-396, 1982.

D’ANDREA, A.; ALIBONI., A.; De SANTIS, A.; MARIANI, S.; GORGOGLIONE, D.; RITIENI, A. SFE of Derris elliptica (Wallich) Benth. roots: Influence of process parameters on yield and purity of rotenone. Journal of Supercritical Fluids, v.42, p. 330-333, 2007.

Del VALLE, J. M.; AGUILERA, J. M. Review: High pressure CO2 extraction. Fundamentals and applications in the food industry, Food Science and Technology International, v.5, p.1-24, 1999.

KING, M. B.; BOTT, T. R. Extraction of Natural Products Using Near-Critical Solvents. Chapman and Hall, Glaskow, UK,p.1-25, 1993.

TAYLOR, L. T. Supercritical Fluid Extraction. John Wiley \& Sons Inc., Canada, p.181, 1996.

TILLY, K. D.; FOSTER, N. R.; MACNAUGHTON, S. J.; TOMASKO, D. L. Viscosity correlations for binary supercritical fluids. Industrial and Engineering Chemistry Research, v.33, n.3, p.681-688, 1994. 